Article

\title{
Effects of the Whole-Body Vibration and Auriculotherapy on the Functionality of Knee Osteoarthritis Individuals
}

\author{
Cristiane Ribeiro Kütter ${ }^{1,2}$, Eloá Moreira-Marconi ${ }^{2,3}{ }^{\circledR}$, Ygor Teixeira-Silva ${ }^{2,4}$, \\ Marcia Cristina Moura-Fernandes ${ }^{2,3}$, Alexandre Gonçalves de Meirelles ${ }^{1,2}$, \\ Mario José dos Santos Pereira ${ }^{2}$, Shyang Chang ${ }^{5}$, José Alexandre Bachur ${ }^{6}$, \\ Laisa Liane Paineiras-Domingos $2,4,7 \mathbb{D}$, Redha Taiar ${ }^{8, * \mathbb{D}}$, Mario Bernardo-Filho ${ }^{2} \mathbb{D}$ and \\ Danúbia da Cunha de Sá-Caputo ${ }^{2,4,6}$ (D)
}

1 Mestrado Profissional em Saúde, Medicina Laboratorial e Tecnologia Forense, Instituto de Biologia Roberto Alcantara Gomes, Universidade do Estado do Rio de Janeiro, Rio de Janeiro, RJ 20950-003, Brazil; crkutter@hotmail.com (C.R.K.); meirelles.ale@gmail.com (A.G.d.M.)

2 Laboratório de Vibrações Mecânicas e Práticas Integrativas e Complementares, Departamento de Biofísica e Biometria, Instituto de Biologia Roberto Alcantara Gomes e Policlínica Américo Piquet Carneiro, Universidade do Estado do Rio de Janeiro, RJ 20950-003, Brazil; eloamarconi@gmail.com (E.M.-M.); silvarogy@hotmail.com (Y.T.-S.); marciafernandesfisio@hotmail.com (M.C.M.-F.); mariojsp@gmail.com (M.J.d.S.P.); laisanit@gmail.com (L.L.P.-D.); bernardofilhom@gmail.com (M.B.-F.); dradanubia@gmail.com (D.d.S.-C.)

3 Programa de Pós-graduação em Fisiopatologia Clínica e Experimental, Faculdade de Ciências Médicas, Universidade do Estado do Rio de Janeiro, Rio de Janeiro, RJ 20.551-030, Brazil

4 Programa de Pós-graduação em Ciências Médicas, Faculdade de Ciências Médicas, Universidade do Estado do Rio de Janeiro, Rio de Janeiro, RJ 20.551-030, Brazil

5 Department of Electrical Engineering, National Tsing Hua University, Hsinchu 30013, Taiwan; shyang@ee.nthu.edu.tw

6 Departamento de Medicina e Fisioterapia, Universidade de Franca, Franca, SP 14401-426, Brazil; jabachur@hotmail.com

7 Departamento de Fisoterapia, Faculdade Bezerra de Araújo, Rio de Janeiro, RJ 23052-180, Brazil

8 GRESPI, Université de Reims, 51100 Reims, France

* Correspondence: redha.taiar@univ-reims.fr

Received: 19 October 2019; Accepted: 25 November 2019; Published: 29 November 2019

Featured Application: The protocol proposed by this study (WBV alone or associated with TA) can be used by health professionals as a non-pharmacological intervention to improve the functionality of individuals with knee osteoarthritis.

\begin{abstract}
Knee osteoarthritis (KOA) is a degenerative disease of the knee joint. This study aims to evaluate the effects of whole-body vibration (WBV), auriculotherapy (AT), and the association of these techniques with the functionality of KOA individuals. Individuals $(n=120)$ were allocated an AT group (GAT), a WBV group (GWBV), an association group (GWBV + AT), and their respective controls (CGAT, CGWBMV, CGWBMV + AT). The WBV intervention was performed with 5-14 Hz in 3 min of working time with 1 min rest. The control group performed the protocol with the vibrating platform (VP) turned off. The AT intervention was performed with adhesive tapes, with seeds placed in the both ears on the Shenmen point, knee joint, and kidney. The control groups had seedless tape placed on both ears. The participants were instructed to press the adhesive tapes with the fingers three times per day (for 6 days) and to remove the adhesive tapes on the seventh day, before returning to the laboratory. The International Knee Documentation Committee (IKDC), the short physical performance battery (SPPB), and the anterior trunk flexibility (ATF) tests were applied. Acute and cumulative effects were determined. In first session (acute effect of the first session), significant
\end{abstract}


improvements were observed in the groups GWBV $(p=0.03)$ and GWBV + AT $(p=0.04)$, and in the cumulative effect a significant improvement was observed in the groups GWBV $(p=0.02)$ and GWBV + AT $(p=0.01)$. Concerning the overall score of the SPPB, significant improvements were observed in the individuals of the GWBV $(p=0.01)$ and GWBV $+\mathrm{AT}(p=0.03)$ groups (cumulative effect). No changes were found in the score for the IKDC. The WBV alone or associated with AT, besides being a safe and feasible strategy, likely produces physiological responses that improve the functionality of KOA individuals, considering the findings of the ATF and the score of the SPPB.

Keywords: mechanical vibration; traditional Chinese medicine; balance; flexibility; muscle strength

\section{Introduction}

Knee osteoarthritis (KOA) is characterized by the degradation and erosion of the articular cartilage, inflammation of the synovial membrane, and formation of osteophytes and ligament laxity $[1,2]$. These conditions could lead to a relevant level of pain, a reduction in the movement of the knees and joint stiffness with commitment of functionality. Ferreira el al. (2018), published an umbrella review, in which it is pointed out that KOA evolution can improve, remain stable, or get worse. Moreover, it is established that treatment strategies include pharmacological, non-pharmacological, surgical, and non-surgical interventions. Furthermore, it is highlighted that there are several non-pharmacological and non-surgical interventions that are safe and with low cost, which can be incorporated into self-management performed at home or in the community, and that have a relevant public health impact [3]. In this context, therapeutic interventions with less side effects for KOA individuals would be desirable, such as various modalities of exercise [4], such as whole-body vibration (WBV) exercises [3,5], physiotherapy techniques, and the procedures related to traditional Chinese medicine (TCM), such as acupuncture [6,7] and auriculotherapy (AT) [5].

One technique among the TCM procedure is AT [8,9]. In TCM, disease is considered to be caused by the imbalance of the energy, qi, in the body [10].

Similar points to acupuncture are also found around areas of the sensory organs, such as on the ears in the AT $[8,11,12]$. AT is a procedure in which there is a stimulation of point (acupoint) on specific areas of the inner and outer ear lobe that treat disease or illness [10].

In AT, different types of materials, including plant seeds (Semen vaccariae), are applied to stimulate the acupoints for therapeutic purposes. The effectiveness of AT would be associated with functional changes in the body, interconnected with TCM, neurological, and embryological theories, microsystems, and a hormonal basis [9,12-15].

In general, AT involves the stimulation of a point named the "corresponding region" related to the organic dysfunction to be treated. Other points are also chosen considering the disease. AT has demonstrated beneficial effects in various clinical disorders $[12,14,16,17]$, such as pain and anxiety [8], pain associated with cancer [18], knee arthroscopy, hip fractures, and hip arthroplasty [19].

An important alternative to physical activity is the WBV exercises, which have been used to improve fitness performance $[20,21]$ or as an option for the rehabilitation process [22]. WBV exercises take place when the individual has direct contact with the base of a vibrating platform (VP). In this case, there is a transmission of the mechanical vibration generated in the VP to the body of the individual $[23,24]$.

Studies involving WBV exercises suggested important findings, such as mass gain and muscle strength, balance, walking speed, decrease of pain [22-24], improvement in flexibility $[5,25,26]$, and a decrease in the risk of falling [27].

Different tools are used to assess the functionality of various populations [28,29], for example, The International Knee Documentation Committee (IKDC) [29]. In addition, a short physical performance battery (SPPB) is used to evaluate the physical performance of individuals and evaluate 
their functional capacity considering balance, gait speed, and lower limb strength [30]. The functionality can also be assessed using the anterior trunk flexibility (ATF) test [25], in which the individual is asked to measure the distance between their middle finger to floor.

Considering the relevance of the use of non-pharmacological procedures to the management of individuals with KOA, the aim of this study was to analyze the effect of WBV and AT and the association of these two techniques on the functionality of KOA individuals. Thus, the hypothesis states that WBV and AT (associated or isolated) could be a safe and feasible strategy for improving the functionality of KOA individuals.

\section{Materials and Methods}

This cross-section and cross-over study was approved by the Ethics Committee in Research of the Hospital Universitário Pedro Ernesto (HUPE) and the Universidade do Estado do Rio de Janeiro (UERJ), and it was registered in the clinical trial registration (CAAE-19826413.8.0000.5259 and RBR-7dfwct, respectively). All the procedures are embedded by the Declaration of Helsinki. The interventions were performed in Laboratório de Vibrações Mecânicas e Práticas Integrativas (LAVIMPI), UERJ, from 2013 to 2019 .

\subsection{Inclusion Criteria}

Participants aged $\geq 40$ years old of both sexes who werr outpatients of the HUPE with a diagnosis of KOA.

\subsection{Exclusion Criteria}

Participants with untreated hypertension, pregnancy, neurological diseases, "fear" of the movements on the VP, neurological alterations, which may hamper understanding of assessment instruments, severe or incapacitating clinical diseases, concomitant musculoskeletal disorders, prosthesis in the knee or hip, criterium of the investigator (aiming for the safety of the participant), and those individuals who refused to sign the Informed Agreement Form to participate in the study.

\subsection{Sample Size}

For a statistical power of $95 \%$ and a significance level of 5\%, a sample size of 13 subjects in each group, according to the variable functionality, was calculated [31]. Considering a possible decrease in sample size, 222 individuals were recruited. The subjects were referred by a physician of the Orthopedics Department of the HUPE/UERJ. The individuals were asked to maintain the regular dietary habits and level of physical activity during the study period.

\subsection{Participants}

A total of 102 individuals were excluded due to the exclusion criteria or economic conditions or because the individual gave up. A total of 120 individuals were allocated into six groups: the AT group (GAT), the WBV group (GWBV), the group with the association of interventions (GWBV + AT), and their respective controls (CGAT, CGWBV, CGWBV + AT). In all the groups there were, at least, two men. In the GWBV and GWBV + AT, the intervention was performed twice a week and, in the GAT, once a week following a previous publication [5]. The control groups performed the same procedure, but with the side alternating vibrating platform (SAVP) turned off.

To try to optimize the study, the allocation of the individuals in each group considered the order of the moment of the arrival in the LAVIMPI. The first individual entered in the GAT, the second in the GWBV, and in sequence up to GWBV + AT, CGAT, CGWBV, and CGWBV + AT. The seventh individual and the next individuals followed a new sequence. 


\subsection{Whole-Body Vibration Intervention}

The 5-week, twice a week protocol used a SAVP (Novaplate Fitness Evolution ${ }^{\circledR}$, Brazil), following a previous publication [5]. In these platforms mechanical vibrations are generated. Mechanical vibrations are physical agents with oscillatory, sinusoidal, and deterministic movements around an equilibrium point $[25,32,33]$. The individual was seated in a chair with a bare foot on the base of the SAVP, with knee flexion and the hands resting on the knees (Figure 1). The biomechanical parameters of the mechanical vibration used in this protocol were peak-to-peak displacement (D) with 2.5, 5.0, and $7.5 \mathrm{~mm}$, peak acceleration from 0.12 up to $2.95 \times \mathrm{g}$. and a working time of $3 \mathrm{~min}$ interspersed by a $1 \mathrm{~min}$ rest. This sequence was applied three times. The frequency varied from $5 \mathrm{~Hz}$ (in the first day) to $14 \mathrm{~Hz}$ (in the last day), increasing by $1 \mathrm{~Hz}$ per session. This protocol followed a previous publication [5].

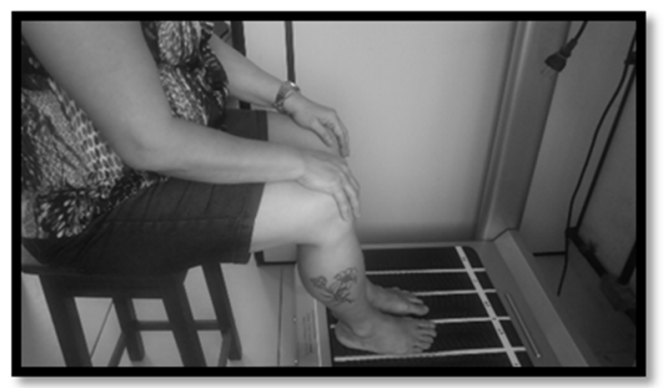

Figure 1. Position of the individual in the auxiliary chair with their feet on the SAVP.

\subsection{Auricular Intervention}

The auricular points were chosen according to AT principles $[12,14,16,17]$. The Shenmen point is an analgesic point, the Knee joint point is the corresponding point, and the kidney has a function related to the bones and lower limbs. The knee joint is located at the superior crus of the antihelix, at the same level of the superior border of the inferior crus of the antihelix. The Shenmen is located at the bifurcation of the crura of the antihelix. The kidney lies in the upper part of the cymba conchae at the superior portion of the acupuncture point of the small intestine [14,15,34].

Adhesive tapes with two mustard (Semen vaccariae) seeds (Figure 2A up) were placed in both ears in referred points (Figure 2A down). The control groups had the seedless (Figure 2B up) tape placed in the lobes of both ears (Figure 2B down).

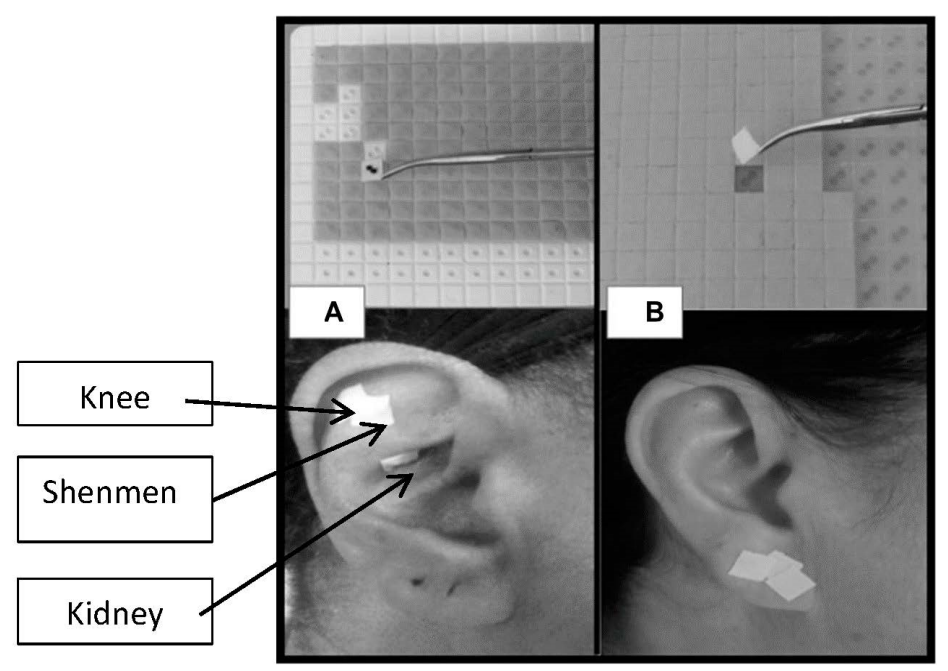

Figure 2. (A) Auriculotherapy (AT) points (knee, kidney, and Shenmen); (B) control group AT.

The participants were instructed to use the fingers to press the adhesive tapes for $10 \mathrm{~min}$, three times per day (six days), and to remove the adhesive tapes on the seventh day, before returning to the 
laboratory. This procedure was done for five weeks. Before the placement of the adhesive tapes, local visual inspection and local asepsis with alcohol at $70 \%$ were performed. Figure 3 shows the steps of the interventions using adhesive tapes in the ears of the participants.

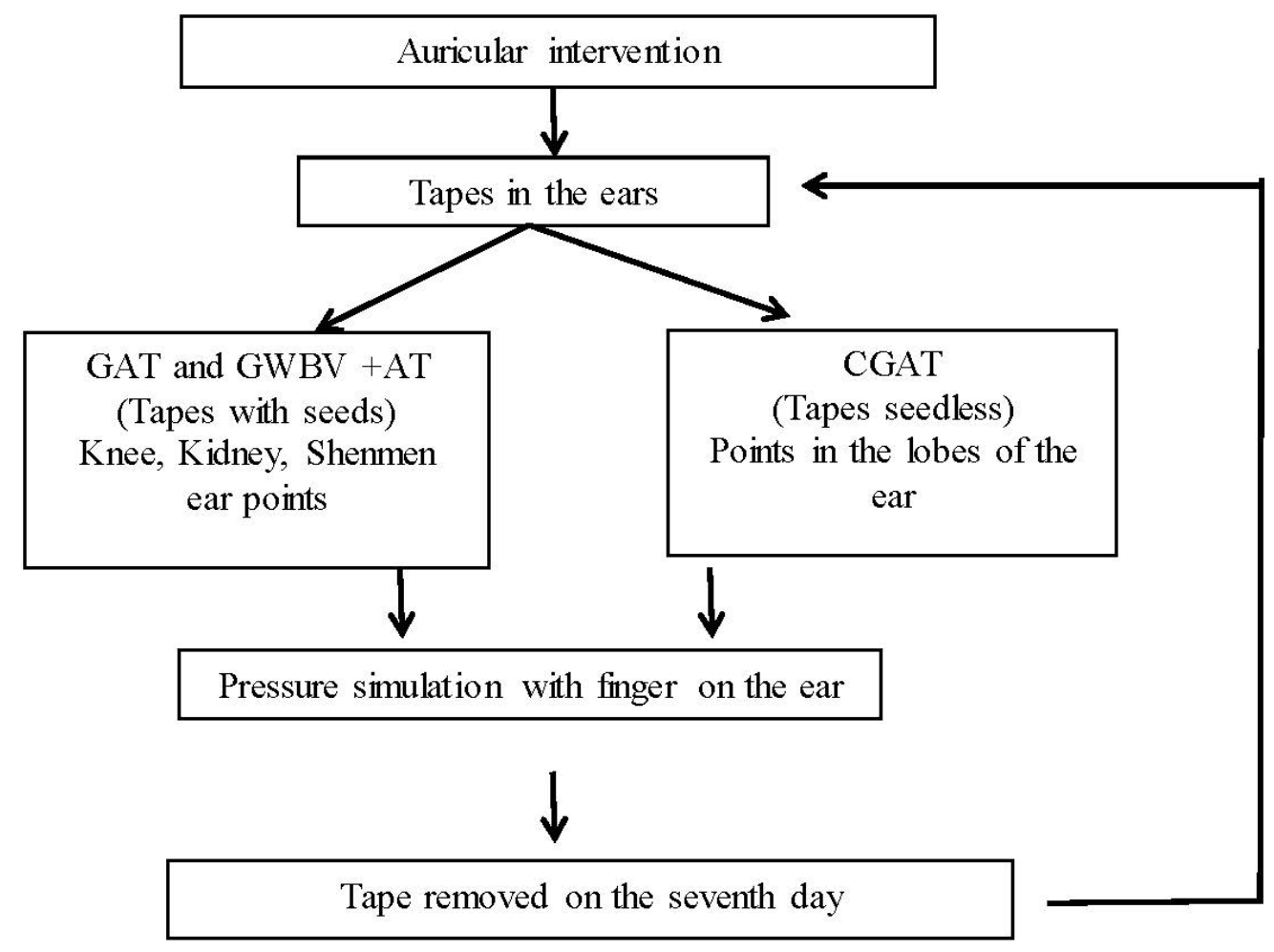

Figure 3. Steps of the interventions using adhesive tapes in the ears of the participants. group (GAT), control of the AT group (CGAT), whole-body vibration group (GWBV) + AT group with WBV and AT associated.

\section{Primary Outcome Measures}

The evaluations of anterior trunk flexion (ATF), the International Knee Documentation Committee (IKDC), and short physical performance battery (SPPB) tests were performed longitudinally, as described in the next paragraphs of the methodology.

\subsection{Anterior Trunk Flexion (ATF)}

The individuals of the all groups performed the ATF, as shown in Figure 4. To measure flexibility, the individual was asked to perform an anterior trunk flexion with the lower limbs extended and with the head relaxed at the maximum flexion. The difference $(\triangle \mathrm{ATF})$ between the measurements before and after the first session (acute effect of the first session) and before and after the last session (acute effect of the last session) were considered. The difference ( $\triangle$ ATF) between the measurements before the first session and after last session (cumulative effect) were also considered [33].

\subsection{International Knee Documentation Committee}

The International Knee Documentation Committee created a subjective from which was validated in Brazil [35] attached as a Supplementary Document to this work. It contains 18 items/questions to verify symptoms, function, and sports activities of the knee joint. The lower patient's score was related to the lower function and the intensity of the symptoms [29]. For the score of the IKDC, " 0 " represents the highest level of symptoms and "10" represents the lowest level of symptoms [29]. This tool was applied before the first session and after the last session (cumulative effect). 


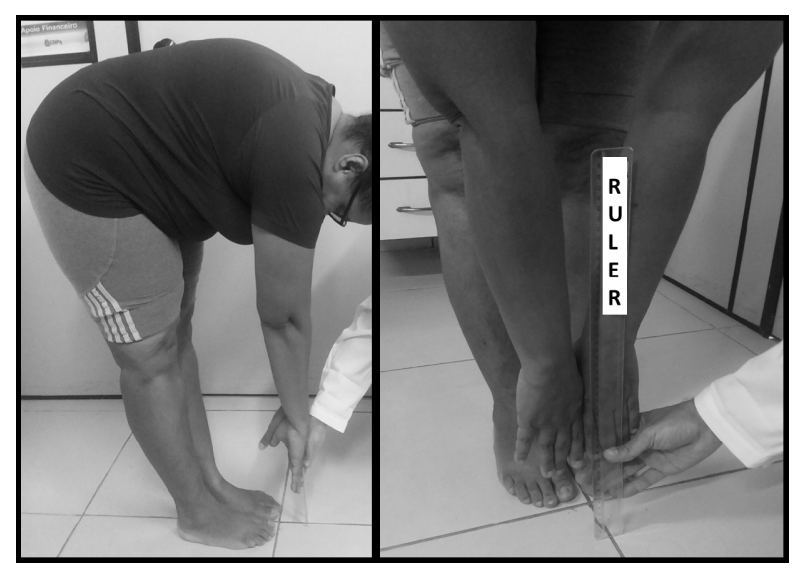

Figure 4. Individual performing anterior trunk flexion.

\subsection{Short Physical Performance Battery (SPPB)}

The SPPB was validated in Brazil and it consists of three tests [30,32]: (a) Balance test: The individual was asked to stay in an appropriated posture for $10 \mathrm{~s}$ in three different positions (feet together, semi-tandem, and tandem); (b) walking time test: The individual was asked to walk two times for $3 \mathrm{~m}$ (marked on the floor) and it was timed, and the shortest time between the two was considered; (c) five times sit-to-stand test: The individual was asked to get up and sit on the chair 5 times, with the arms crossed on the chest, as fast as possible (timed). This tool was applied before the first session and after the last session (cumulative effect).

\section{Statistical Analysis}

The Shapiro-Wilk test was used to verify the normality of the data. The differences between the intra-groups was analyzed using the Wilcoxon rank test. The comparisons between the findings of the intervention group and the respective control group was carried out with the Mann-Whitney test. Kruskal Wallis and student Newman-Keuls tests were used to compare the anthropometric characteristics of all the groups. The value used was $p \leq 0.05$ as the significant. The software used was BioEstat 5.3.

\section{Results}

The enrollment of the participants in the study is shown in the Figure 5 in the flow diagram. Individuals $(\mathrm{n}=222)$ were recruited and 102 were eliminated from the work (due to exclusion criteria or economic conditions or because the individual gave up). Consequently, 120 individuals (106 women and 14 men) participated in this study. They were allocated in the AT group (GAT), the WBV group (GWBV), and the group with the association of interventions (GWBV + AT) and their respective controls (CGAT, CGWBV, and CGWBV + AT). The number of individuals that performed the evaluations (ATF, IKDC, and SPPB) is also indicated.

Statistical analyses of some anthropometric parameters (age, stature, and body mass) of the individuals in the various groups was performed. Although only three anthropometric characteristics were considered, the results in Table 1 indicate no difference among the groups $(p>0.05)$. This finding shows a homogeneity among the groups. 


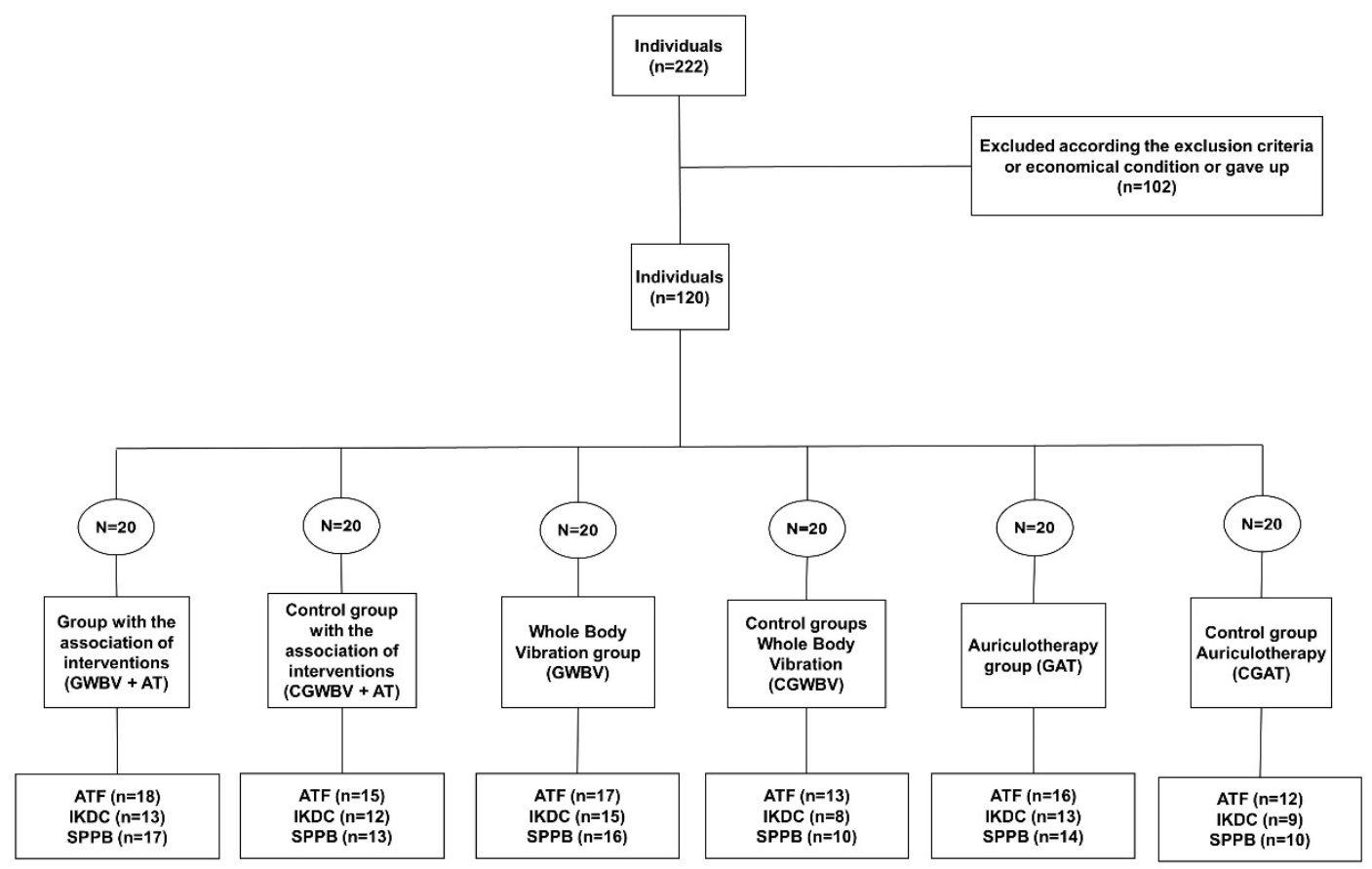

ATF - Anterior Trunk Flexion, IKDC - International Knee Documentation Committee, SPPB- Short Physical Performance Battery

Figure 5. Enrollment of the participants in the study.

Table 1. Anthropometric characteristics of the individuals included in the work.

\begin{tabular}{cccc}
\hline Groups & Age (Year) $\mathbf{( M d} \pm \mathbf{S D})$ & Stature (m) $\mathbf{( M d} \pm \mathbf{S D})$ & $\begin{array}{c}\text { Body Mass (kg) } \\
\mathbf{( M d} \pm \mathbf{S D})\end{array}$ \\
\hline CGAT & $63.00 \pm 7.00$ & $1.60 \pm 0.08$ & $85.67 \pm 14.98$ \\
GAT & $64.00 \pm 7.00$ & $1.59 \pm 0.09$ & $84.58 \pm 14.90$ \\
CGWBV & $66.00 \pm 12.00$ & $1.57 \pm 0.07$ & $84.50 \pm 13.38$ \\
GWBV & $67.00 \pm 10.00$ & $1.57 \pm 0.05$ & $84.59 \pm 14.15$ \\
CGWBV + AT & $65.00 \pm 7.00$ & $1.55 \pm 0.04$ & $84.96 \pm 14.59$ \\
GWBV + AT & $65.00 \pm 8.00$ & $1.55 \pm 0.08$ & $85.49 \pm 14.36$ \\
$p$-value & 0.852 & 0.310 & 0.999 \\
\hline
\end{tabular}

Media (Md), standard deviation (SD), meter (m), kilogram (kg), control group auriculotherapy group (CGAT), auriculotherapy group (GAT), control whole-body vibration group (CGWBV), whole-body vibration group (GWBV), control whole-body vibration group and auriculotherapy (CGWBV + AT), whole-body vibration group and auriculotherapy $(\mathrm{GWBV}+\mathrm{AT})$, Kruskal Wallis and student Newman-Keuls tests, level of significance $p \leq 0.05$.

The $\triangle$ ATF was calculated considering the measurements before and after the first session (acute effect of the first session), and significant improvements were observed in the groups GWBV $(p=0.03)$ and GWBV + AT ( $p=0.04)$ (Figure 6A).

The $\triangle$ ATF was calculated considering the measurements before and after the last session, and there were no significant changes (acute effect) (Figure 6B).

The $\triangle$ ATF was calculated considering the measurements before the first session and after the last session (cumulative effect), and significant improvements were observed in the groups GWBV ( $p=$ $0.02)$ and GWBV + AT ( $p=0.01)$ (Figure 6C).

The findings concerning the IKDC (for all groups) are presented in Table 2. The analysis of the results shows no significant changes $(p>0.05)$ among all the groups and respective controls. 

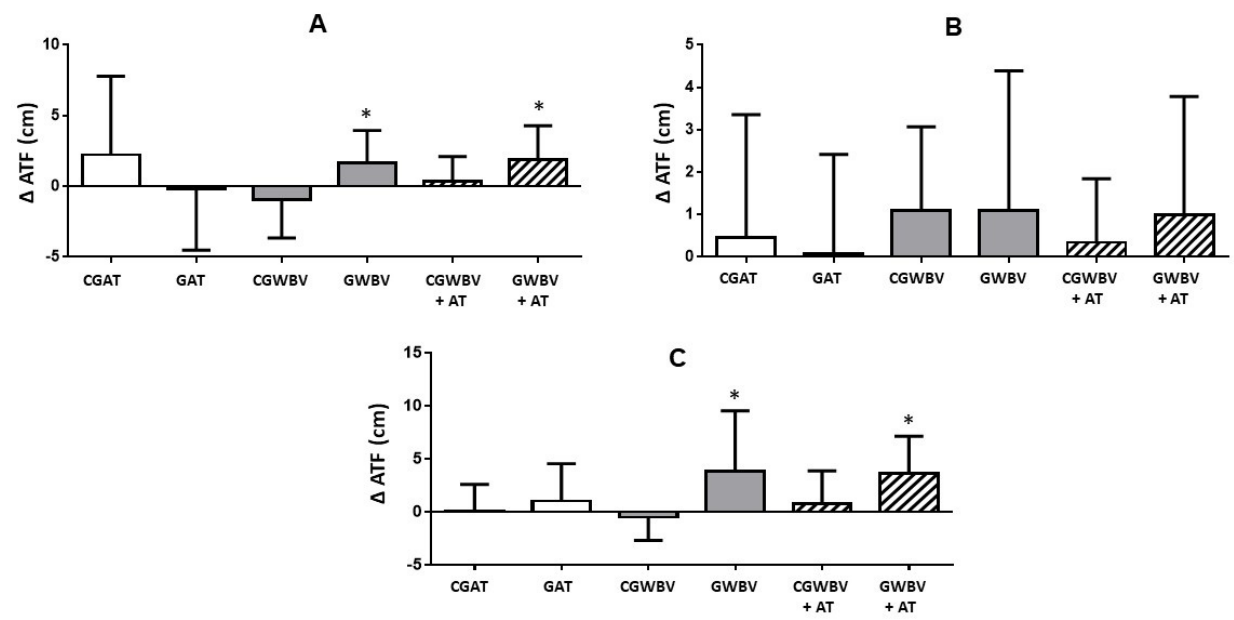

Figure 6. The findings concerning the anterior trunk flexion (ATF) test of all groups. Control auriculotherapy group (CGAT), auriculotherapy group (GAT), control whole-body vibration group (CGWBV), whole-body vibration group (GWBV), control whole-body vibration group and auriculotherapy $(\mathrm{CGWBV}+\mathrm{AT})$, whole-body vibration group and auriculotherapy (GWBV + AT). (A) The acute effect of the first session. GWBV $(p=0.03)$ and GWBV + AT $(p=0.04)$. (B) The acute effect of the last session. (C) The cumulative effect. GWBV $(p=0.02)$ and GWBV + AT $(p=0.01)$.

Table 2. The data concerning the International Knee Documentation Committee score.

\begin{tabular}{ccccccc}
\hline & \multicolumn{3}{c}{ Control } & & \multicolumn{3}{c}{ Intervention } \\
\hline & Before & After & & Before & After & \\
\hline & Md $\pm \mathrm{SD}$ & $\mathrm{Md} \pm \mathrm{SD}$ & $p$-value* & $\mathrm{Md} \pm \mathrm{SD}$ & $\mathrm{Md} \pm \mathrm{SD}$ & $p$-value* \\
& $/ 100$ & $/ 100$ & & $/ 100$ & $/ 100$ & \\
GAT & $24.68 \pm 9.85$ & $27.58 \pm 9.71$ & 0.098 & $33.59 \pm 16.84$ & $35.91 \pm 17.70$ & 0.37 \\
GWBV & $28.87 \pm 13.69$ & $34.19 \pm 11.45$ & 0.299 & $31.55 \pm 13.21$ & $33.79 \pm 9.07$ & 0.43 \\
GWBV + AT & $27.07 \pm 7.86$ & $30.26 \pm 8.42$ & 0.059 & $31.03 \pm 11.85$ & $34.49 \pm 14.67$ & 0.14 \\
\hline
\end{tabular}

Media, (Md) standard deviation (SD), auriculotherapy group (GAT), whole-body vibration group (GWBV), whole-body vibration group and auriculotherapy (GWBV + AT), $p$-value, level of significance $p \leq 0.05^{*}$.

The findings concerning the overall score of SPPB involving all groups are presented in the Table 3. Significant changes $(p \leq 0.05)$ were observed in the individuals of the GWBV and GWBV + AT groups (the cumulative effect). No significant changes $(p>0.05)$ were verified in the GAT and in the control groups.

Table 3. The data concerning the short physical performance battery (SPPB) overall score.

\begin{tabular}{ccccccc}
\hline & \multicolumn{3}{c}{ Control } & \multicolumn{3}{c}{ Intervention } \\
\hline & Before & After & & Before & After & \\
\hline & $\begin{array}{c}\text { Med } \pm \text { IQR } \\
\text { (score) }\end{array}$ & $\begin{array}{c}\text { Med } \pm \text { IQR } \\
\text { (score) }\end{array}$ & $p$-value* & $\begin{array}{c}\text { Med } \pm \text { IQR } \\
\text { (score) }\end{array}$ & $\begin{array}{c}\text { Med } \pm \text { IQR } \\
(\text { score })\end{array}$ & $p$-value* \\
GAT & $7.00 \pm 1.75$ & $7.00 \pm 2.00$ & 0.529 & $7.00 \pm 3.75$ & $7.50 \pm 2.00$ & 0.29 \\
GWBV & $7.00 \pm 2.00$ & $7.00 \pm 7.00$ & 0.798 & $7.00 \pm 2.00$ & $8.00 \pm 2.00$ & $0.01^{*}$ \\
GWBV + AT & $7.00 \pm 4.00$ & $7.00 \pm 3.00$ & 0.834 & $7.00 \pm 1.50$ & $8.50 \pm 1.75$ & 0.03 \\
\hline
\end{tabular}

Median (Med), interquartile range (IQR), auriculotherapy group (GAT), whole-body vibration group (GWBV), whole-body vibration group and auriculotherapy (GWBV + AT), $p$-value, level of significance $p \leq 0.05^{*}$.

\section{Discussion}

As hypothesized, the current work has demonstrated that WBV alone, or in association with AT, improves the functionality of KOA individuals, considering the ATF and overall score of the SPPB. 
However, these improvements were not associated with the responses in the IKDC score. A suggestion might be associated with a subjective approach of the IKDC.

Costa et al. (2014) [23] described, in a systematic review, the different treatments used on the management of the individuals with osteoarthritis and the importance of the WBV exercise as a modality of exercise in this context. Moreover, Ferreira et al. (2018) [3] pointed out that there are several non-pharmacological and non-surgical interventions, including WBV and acupuncture, for the management of KOA individuals. Suen et al. (2016) [13] showed the effect of the AT with and without magnetotherapy in KOA individuals and observed improvements in their functionality. Costa-Cavalcanti and colleagues [36] evaluated the effect of auriculotherapy on biochemical parameters during 5 weeks, once a week. The study showed a significant reduction in direct bilirubin, aspartate aminotransferase, and triglycerides.

Considering the ATF, significant changes (improvements) were observed with the intervention of WBV alone or with the AT (acute and cumulative effect) (Figure 6A,C). In the acute effect of the last session, alterations were not found in the ATF, likely because the individuals had already reached the maximum of answers for the stimulus in the previous sessions (Figure 6B). These findings are in agreement with Sá-Caputo and colleagues [25,33], who showed improvements in the flexibility after the WBV exercise protocol in individuals with metabolic syndrome. Neto and colleagues [5] reported a significant decrease of the level of pain in the KOA individuals treated with WBV alone and with a combined intervention (WBV and AT). In addition, Moreira-Marconi and colleagues [37] reported that WBV alone, and with a combined intervention with AT, improved handgrip strength in cumulative response. These responses [25,33] could favor the findings presented in Figure 6A,B related to the ATF. The absence of the effect of the interventions in the acute effect of the last session could be associated with the adaptation phenomenon to the stimulus [38] and/or genetic characteristics of the individuals to the response to the stimulus [39]. These facts could justify the uncertainty of the findings of Figure $6 \mathrm{~B}$ with elevated standard deviations of the measurements.

Using the protocol of the current study, for five weeks, once (AT) or twice (AT and WBV or only WBV) per week, no difference was observed in any group using the IKDC score (Table 2). Park and colleagues [40] described improvements related with the use of the Korean Western Ontario McMaster score (KWOMAC) in KOA individuals, who performed the WBV exercise for 8 weeks, three times per week. These differences could be associated with the protocol used in this study (five weeks/once or twice a week) whereas in the other study [40] the intervention was 8 weeks/three times a week. Moreover, there is a difference in the qualitative tools used in the evaluation, IKDC and KWOMAC.

Concerning the functionality (considering the SPPB) of the KOA individuals, in the cumulative effects, an improvement was found in the interventions with WBV alone and WBV and AT (Table 3). This finding is in agreement with Trans et al. (2009) [41] in a protocol of the WBV exercise twice a week for 8 weeks, with a progressive increase intensity (frequencies among $25-30 \mathrm{~Hz}$ ), which performed unloaded static WBV exercise. They concluded that WBV is a safe method for rehabilitation of women with KOA, increasing the muscle strength. Wang et al., 2016 [42] showed improvements in the symptoms, physical function, and spatiotemporal parameters with a protocol of the WBV exercise using a frequency of $35 \mathrm{~Hz}$ and peak-to-peak displacement of 4-6 $\mathrm{mm}$ for 5 days a week for 12 weeks in a vertical VP. Avelar et al. (2011) [31] described that the addition of the WBV exercise to squat training failed to result in a significant improvement in functional performance and self-reported status of knee osteoarthritis in the elderly. However, the intragroup results suggest that the WBV exercise may present a feasible and effective way of improving the functionality and self-perception of the disease status in older adults with KOA. They used frequencies from $25-40 \mathrm{~Hz}$ and peak-to-peak displacement of $4 \mathrm{~mm}$ for 12 weeks in a vertical VP. The improvement observed on the functional performance could be associated with muscle stimulation due to exposition to mechanical vibration in the WBV exercise. Paineiras-Domingos et al. (2018) [32] found significant responses in the improvement of the functionality of Metabolic Sindrome individuals that performed WBV exercises, analyzing the overall score of the SPPB and the three tests (balance test, the gait speed, and the five chair stand test). 
In the current study, an alternative unusual position of the patient was used, compared to other studies [5,41,42], involving the WBV exercise in KOA individuals. The selection of this position (seated in a chair, barefoot, on the basis of the SAVP) is related to reduction of the load and the impact in the knee joint so as to make these individuals able to practice the exercise [5].

An important finding of this investigation was the absence of any undesirable effect due to the interventions, either isolated (WBV or AT) or combined (WBV and AT). These considerations reinforce the relevance of these procedures, as useful, safe, and feasible. Moreover, they are inexpensive.

This study had some limitations and can be improved, such as the total time (5 weeks) of the protocol of the WBV exercise. The elevated ratio between females and males in the groups was also a limitation. Only three anthropometric characteristics of the participants were evaluated. Another limitation was that no specific tool was used to evaluate the level of physical activity of the KOA individuals. Concerning the AT interventions, only three points of the ear were stimulated. In addition, temporal analysis of the responses in the various sessions was not done. These considerations could help to guide future experimental designs.

\section{Conclusions}

In spite of the limitations, it is still possible to conclude that the WBV alone or associated with AT might induce physiological responses that favour the improvement of the flexibility with the evaluation of anterior flexibility of the trunk and the functionality (SPPB-overall score) of the KOA individuals. Moreover, the absence of effects of these interventions in the acute response of the last session could be associated with possible adaptation and/or genetic characteristics of the studied population. In consequence, a temporal analysis of these responses in the various sessions and the addition of other quantitative parameters in the protocols are ongoing.

Supplementary Materials: The following are available online at http://www.mdpi.com/2076-3417/9/23/5194/s1.

Author Contributions: Conceptualization, M.B.-F. and D.d.C.d.S.-C.; data curation, C.R.K., E.M.-M., Y.T.-S., M.C.M.-F., A.G.d.M., M.J.d.S.P., and L.L.P.-D.; formal analysis, M.J.d.S.P.; funding acquisition, M.B.-F.; investigation, C.R.K., E.M.-M., Y.T.-S., M.C.M.-F., A.G.d.M., and L.L.P.-D.; methodology, C.R.K., E.M.-M., Y.T.-S., M.C.M.-F., A.G.d.M., and L.L.P.-D.; project administration, D.d.C.d.S.-C.; writing-original draft, C.R.K. and M.B.-F.; writing-review and editing, S.C., J.A.B., R.T., and D.d.C.d.S.-C.

Funding: This research was funded by Coordenação de Aperfeiçoamento de Pessoal de Nível Superior-Brazil (CAPES)-Finance Code 001, the CNPq (Conselho Nacional de Desenvolvimento Científico e Tecnológico), and FAPERJ (Fundação Carlos Chagas Filho de Amparo à Pesquisa do Estado do Rio de Janeiro).

Conflicts of Interest: The authors declare no conflict of interest.

\section{References}

1. Hochberg, M.C.; Altman, R.D.; April, K.T.; Benkhalti, M.; Guyatt, G.; McGowan, J.; Towheed, T.; Welch, V.; Wells, G.; Tugwell, P. American College of Rheumatology 2012 recommendations for the use of nonpharmacologic and pharmacologic therapies in osteoarthritis of the hand, hip, and knee. Arthritis Care Res. 2012, 64, 465-474. [CrossRef] [PubMed]

2. Dulay, G.S.; Cooper, C.; Dennison, E.M. Knee pain, knee injury, knee osteoarthritis \& work. Best Pract. Res. Clin. Rheumatol. 2015, 29, 454-461. [PubMed]

3. Ferreira, R.; Duarte, J.; Gonçalves, R. Non-pharmacological and non-surgical interventions to manage patients with knee osteoarthritis: An umbrella review. Acta Reumatol. Port. 2018, 43, 182-200. [PubMed]

4. Zhang, W.; Moskowitz, R.W.; Nuki, G.; Abramson, S.; Altman, R.D.; Arden, N.; Bierma-Zeinstra, S.; Brandt, K.D.; Croft, P.; Doherty, M.; et al. OARSI recommendations for the management of hip and knee osteoarthritis, Part II: OARSI evidence-based, expert consensus guidelines. Osteoarthr. Cartil. 2008, 16, 137-162. [CrossRef]

5. Neto, S.B.S.; Marconi, E.M.; Kutter, C.R.; Frederico, E.H.F.F.; de Paiva, P.d.C.; Meyer, P.F.; Chang, S.; Sá-Caputo, D.; Bernardo-Filho, M. Beneficial effects of whole body mechanical vibration alone or combined with auriculotherapy in the pain and in flexion of knee of individuals with knee osteoarthritis. Acupunct. Electrother. Res. 2017, 42, 185-201. [CrossRef] 
6. ACR. ACR OA Guidelines Non-Pharmacological-Knee and Hip; American College of Rheumatology: Chicago, IL, USA, 2009; pp. 4-30.

7. Allen, K.D.; Bierma-Zeinstra, S.M.A.; Foster, N.E.; Golightly, Y.M.; Hawker, G. OARSI Clinical Trials Recommendations: Design and conduct ofimplementation trials of interventions for osteoarthritis. Osteoarthr. Cartil. 2015, 23, 826-838. [CrossRef]

8. Asher, G.N.; Jonas, D.E.; Coeytaux, R.R.; Reilly, A.C.; Loh, Y.L.; Motsinger-Reif, A.A.; Winham, S.J. Auriculotherapy for Pain Management: A Systematic Review and Meta-Analysis of Randomized Controlled Trials. J. Altern. Complement. Med. 2010, 16, 1097-1108. [CrossRef]

9. Hou, P.W.; Hsu, H.C.; Lin, Y.W.; Tang, N.Y.; Cheng, C.Y.; Hsieh, C.L. The history, mechanism, and clinical application of auricular therapy in traditional Chinese medicine. Evid.-Based Complement. Altern. Med. 2015, 2015, 495684. [CrossRef]

10. Huang, L. Auricular Medicine: A Complete Manual of Auricular Diagnosis and Treatment; Auricular International Research and Training: Orlando, FL, USA, 2005.

11. Rabischong, P.; Terral, C. Scientific Basis of Auriculotherapy: State of the Art. Med. Acupunct. 2014, 26, 84-96. [CrossRef]

12. Suen, L.K.P.; Wong, T.K.S.; Leung, A.W.N. Is there a place for auricular therapy in the realm of nursing? Complement. Ther. Nurs. Midwifery 2001, 7, 132-139. [CrossRef]

13. Suen, L.K.P.; Yeh, C.H.; Yeung, S.K.W. Erratum to Using auriculotherapy for osteoarthritic knee among elders: A double-blinded randomised feasibility study. BMC Complement. Altern. Med. 2016, 16, 257. [CrossRef] [PubMed]

14. Round, R.; Litscher, G.; Bahr, F. Auricular acupuncture with laser. Evid.-Based Complement. Altern. Med. 2013, 2013, 984763. [CrossRef] [PubMed]

15. Rubach, A. Principles of Ear Acupuncture-Microsystem of the Auricle: Microsystem of the Auricle, 2nd ed.; Thieme Publishers: Stuttgart, Germany, 2001.

16. Gori, L.; Firenzuoli, F. Ear acupuncture in European traditional medicine. Evid.-Based Complement. Altern. Med. 2007, 4, 13-16. [CrossRef] [PubMed]

17. Suen, L.K.P.; Wong, T.K.S.; Leung, A.W.N. Auricular therapy using magnetic pearls on sleep: A standardized protocol for the elderly with insomnia. Clin. Acupunct. Orient. Med. 2002, 3, 39-50. [CrossRef]

18. Alimi, D.; Rubino, C.; Pichard-Léandri, E.; Fermand-Brulé, S.; Dubreuil-Lemaire, M.L.; Hill, C. Analgesic effect of auricular acupuncture for cancer pain: A randomized, blinded, controlled trial. J. Clin. Oncol. 2003, 21, 4120-4126. [CrossRef]

19. Usichenko, T.I.; Dinse, M.; Hermsen, M.; Witstruck, T.; Pavlovic, D.; Lehmann, C. Auricular acupuncture for pain relief after total hip arthroplasty-A randomized controlled study. Pain 2005, 114, 320-327. [CrossRef]

20. Sitjà-Rabert, M.; Rigau, D.; Fort Vanmeerghaeghe, A.; Romero-Rodríguez, D.; Bonastre Subirana, M.; Bonfill, X . Efficacy of whole body vibration exercise in older people: A systematic review. Disabil. Rehabil. 2012, 34, 883-893. [CrossRef]

21. Rittweger, J. Vibration as an exercise modality: How it may work, and what its potential might be. Eur. J. Appl. Physiol. 2010, 108, 877-904. [CrossRef]

22. Rauch, F.; Sievanen, H.; Boonen, S.; Cardinale, M.; Degens, H.; Felsenberg, D.; Roth, J.; Schoenau, E.; Verschueren, S.; Rittweger, J. Reporting whole-body vibration intervention studies: Recommendations of the International Society of Musculoskeletal and Neuronal Interactions. J. Musculoskelet. Neuronal Interact. 2010, 10, 193-198.

23. da Costa, P.R.; da Cunha S\&a-Caputo, D.U.; Arn\&obio, A.; Pacheco, R.; Kutter, C.; Costa, R.; Giehl, P.M.; Paiva, D.N.; Marin, P.J.; Salmon, J.R.; et al. Whole-body vibration and benefits for people with osteoarthritis: A systematic review. Int. J. Med. Med. Sci. 2014, 6, 201-210.

24. Di Giminiani, R.; Manno, R.; Scrimaglio, R.; Sementilli, G.; Tihanyi, J. Effects of individualized whole-body vibration on muscle flexibility and mechanical power. J. Sports Med. 2010, 50, 139-151.

25. Sá-Caputo, D.D.; Ronikeili-Costa, P.; Carvalho-Lima, R.P.; Bernardo, L.C.; Bravo-Monteiro, M.O.; Costa, R.; Moraes-Silva, J.D.; Paiva, D.N.; Machado, C.B.; Mantilla-Giehl, P.; et al. Whole Body Vibration Exercises and the Improvement of the Flexibility in Patient with Metabolic Syndrome. J. Rehabil. Res. Dev. 2014, 2014, 628518. [CrossRef] [PubMed]

26. Yang, J.; Seo, D. The effects of whole body vibration on static balance, spinal curvature, pain, and disability of patients with low back pain. J. Phys. Ther. Sci. 2015, 27, 805-808. [CrossRef] 
27. Wang, P.; Yang, X.; Yang, Y.; Yang, L.; Zhou, Y.; Liu, C.; Reinhardt, J.D.; He, C. Effects of whole body vibration on pain, stiffness and physical functions in patients with knee osteoarthritis: A systematic review and meta-analysis. Clin. Rehabil. 2015, 29, 939-951. [CrossRef] [PubMed]

28. Collins, N.J.; Misra, D. Measures of Knee Function. Arthritis Care Res. 2011, 63, 208-228. [CrossRef] [PubMed]

29. Marshall, C.J.; Dobson, F.; Hinman, R.S.; Sayer, T.; Anderson, C.; Newcomb, N.; Bennell, K.L. Reliability of the osteoarthritis research society international recommended performance-based tests of physical function in people with hip and knee osteoarthritis. Osteoarthr. Cartil. 2015, 23, A343-A344. [CrossRef]

30. Bennell, K.; Dobson, F.; Hinman, R. Measures of physical performance assessments: Self-Paced Walk Test (SPWT), Stair Climb Test (SCT), Six-Minute Walk Test (6MWT), Chair Stand Test (CST), Timed Up \& Go (TUG), Sock Test, Lift and Carry Test (LCT), and Car Task. Arthritis Care Res. 2011, 63, S350-S370.

31. Avelar, N.C.P.; Simão, A.P.; Tossige-Gomes, R.; Neves, C.D.C.; Rocha-Vieira, E.; Coimbra, C.C.; Lacerda, A.C.R. The Effect of Adding Whole-Body Vibration to Squat Training on the Functional Performance and Self-Report of Disease Status in Elderly Patients with Knee Osteoarthritis: A Randomized, Controlled Clinical Study. J. Altern. Complement. Med. 2011, 17, 1149-1155. [CrossRef]

32. Paineiras-Domingos, L.L.; da Cunha Sá-Caputo, D.; Reis, A.S.; Francisca Santos, A.; Sousa-Gonçalves, C.R.; Dos Anjos, E.M.; dos Santos Pereira, M.J.; Sartorio, A.; Bernardo-Filho, M. Assessment through the short physical performance battery of the functionality in individuals with metabolic syndrome exposed to whole-body vibration exercises. Dose-Response 2018, 16. [CrossRef]

33. Sá-Caputo, D.C.; Paineiras-Domingos, L.L.; Oliveira, R.; Neves, M.F.T.; Brandão, A.; Marin, P.J.; Sañudo, B.; Furness, T.; Taiar, R.; Bernardo-Filho, M. Acute Effects of Whole-Body Vibration on the Pain Level, Flexibility, and Cardiovascular Responses in Individuals With Metabolic Syndrome. Dose-Response 2018, 16. [CrossRef]

34. Nogier, R. Auriculotherapy; Ed.Thieme: Stuttgart, Germany; New York, NY, USA, 2009; Volume 14.

35. Metsavaht, L.; Leporace, G.; Riberto, M.; de Mello Sposito, M.M.; Batista, L.A. Translation and Cross-Cultural Adaptation of the Brazilian Version of the International Knee Documentation Committee Subjective Knee Form. Am. J. Sports Med. 2010, 38, 1894-1899. [CrossRef] [PubMed]

36. Costa-Cavalcanti, R.G.; de Sá-Caputo, D.D.; Moreira-Marconi, E.; Küter, C.R.; Brandão-Sobrinho-Neto, S.; Paineiras-Domingos, L.L.; Moura-Fernandes, M.C.; da Costa, J.M.; de Moraes Carmo, J.M.; Nogueira-Neto, J.F.; et al. Effect of Auriculotherapy on the Plasma Concentration of Biomarkers in Individuals with Knee Osteoarthritis. J. Acupunct. Meridian Stud. 2018, 11, 145-152. [CrossRef] [PubMed]

37. Moreira-Marconi, E.; Dionello, C.F.; Morel, D.S.; Sá-Caputo, D.C.; Sousa-gonçalves, C.R.; José, M.; Bernardo-Filho, M. Whole body vibration and auriculotherapy improve handgrip strength in individuals with knee osteoarthritis. J. Tradit. Chin. Med. 2019, 39, 707-715.

38. Coffey, V.G.; Hawley, J.A. Concurrent exercise training: Do opposites distract? J. Physiol. 2017, 595, $2883-2896$. [CrossRef]

39. Mann, T.N.; Lamberts, R.P.; Lambert, M.I. High Responders and Low Responders: Factors Associated with Individual Variation in Response to Standardized Training. Sport. Med. 2014, 44, 1113-1124. [CrossRef]

40. Park, Y.G.; Kwon, S.; Park, J.-W.; Cha, D.Y.; Nam, K.Y.; Sim, K.B.; Chang, J.; Lee, H.J. Therapeutic Effect of Whole Body Vibration on Chronic Knee Osteoarthritis. Ann. Rehabil. Med. 2013, 37, 505-515. [CrossRef]

41. Trans, T.; Aaboe, J.; Henriksen, M.; Christensen, R.; Bliddal, H.; Lund, H. Effect of whole body vibration exercise on muscle strength and proprioception in females with knee osteoarthritis. Knee 2009, 16, $256-261$. [CrossRef]

42. Wang, P.; Yang, L.; Li, H.; Lei, Z.; Yang, X.; Liu, C.; Jiang, H.; Zhang, L.; Zhou, Z.; Reinhardt, J.D.D.; et al. Effects of whole-body vibration training with quadriceps strengthening exercise on functioning and gait parameters in patients with medial compartment knee osteoarthritis: A randomised controlled preliminary study. Physiotherapy 2016, 102, 86-92. [CrossRef]

(C) 2019 by the authors. Licensee MDPI, Basel, Switzerland. This article is an open access article distributed under the terms and conditions of the Creative Commons Attribution (CC BY) license (http://creativecommons.org/licenses/by/4.0/). 